Hydroécol. Appl. (1994) Tome 6 Vol 1-2, pp. 115-138

\title{
Bilan du phosphore total dans la retenue de Pareloup
}

\section{Total phosphorus budget in Pareloup reservoir}

\section{F. Sivadier", J.-M. Thébault" et M.-J. Salençon"*}

- Laboratoire d'Hydrobiologie, U.R.A. 695 C.N.R.S., Université Paul Sabatier, 118 route de Narbonne, 31062 Toulouse Cedex, France.

" Electricité de France, Direction des Etudes et Recherches, Département Environnement, 6, quai Watier, 78401 Chatou Cedex, France.

Résumé. - Un bilan du phosphore total dans la retenue de Pareloup a été calculé pour 1986 et 1987.

Les apports de phosphore dans la retenue sont estimés à $29 \mathrm{t}$ entre le $1^{\text {er }}$ avril 1986 et le 31 juillet 1987. Ils ont, comme principale origine, le pompage provenant de la retenue de Bage, sauf en été où les apports par la pollution liée au tourisme prédominent. Les concentrations en phosphore mesurées dans la retenue sont beaucoup plus faibles que celles prévues, à partir des charges annuelles, par les modèles de Vollenweider (1976), Canfield et Bachman (1981) et OCDE (1982). Ces modèles ne semblent pas adaptés à la retenue de Pareloup. La gestion hydraulique ne permet pas de satisfaire une des conditions d'application qui suppose la régularité des apports. De plus, la sédimentation rapide du phosphore provenant de Bage et la disparition des diatomées printanières immobilisent une grande partie du phosphore du système.

Les quantités de phosphore sédimenté pendant la période d'étude, calculées à partir du bilan des entrées et des sorties et des teneurs en phosphore de la retenue, sont évaluées à $90 \%$ du phosphore entrant. Ces valeurs sont en bon accord avec les quantités recueillies dans des pièges à sédiment.

Mots clés. - Phosphore, bilan entrées-sorties, sédimentation.

Abstract. - The total phosphorus budget for Pareloup reservoir was calculated for the years 1986 and 1987.

Phosphorus input in the reservoir was estimated at $29 \mathrm{t}$ for the period between April 1 , 1986 and July 31, 1987. The principal origin is water pumped from Bage reservoir, except in summer, when inputs due to tourism-related pollution are predominant. The phosphorus concentrations actually measured in the reservoir were far lower than predicted, on the basis of annual loading, by the Vollenweider (1976), Canfield and Bachman 
(1981) and OECD (1982) models. These models therefore do not appear well suited to Pareloup. The hydraulic management mode here does not satisfy one of the conditions for their application, which is regularity of inflow concentrations. Moreover, the rapid sedimentation of phosphorus from Bage and the disappearance of spring diatoms immobilize a large proportion of the phosphorus in the system.

The amounts of phosphorus sedimented during the study period, calculated from the balance between the inflow and outflow and phosphorus concentrations in the reservoir, are estimated at $90 \%$ of all phosphorus entering the reservoir. These values agree well with the amounts collected in sediment traps.

Key words. - Phosphorus, input-output budget, sedimentation.

\section{INTRODUCTION}

Dans une grande majorité de lacs naturels et artificiels des régions tempérées, il existe une corrélation entre la biomasse de phytoplancton et le stock de phosphore total disponible. Ce stock résulte d'un bilan entre les apports de phosphore dans la zone pélagique (apports par les affluents et relargage de phosphore par les sédiments) et les retraits (exportation à l'émissaire et sédimentation). Aussi est-il important de quantifier le phosphore des differents apports (affluents, ruissellement, pompage) ainsi que celui des rejets à l'aval (turbinage, débit réservé). L'objectif de ce travail est de dresser le bilan du phosphore total dans la retenue de Pareloup pour 1986 et 1987. L'importante base de données constituée durant ces deux années permettra de calculer dans un premier temps un bilan d'eau équilibré, condition nécessaire à l'élaboration du bilan de phosphore qui pourra ensuite être estimé. La différence entre le phosphore importé, exporté et mesuré dans la retenue constitue une estimation de la sédi- mentation qui sera comparée aux mesures provenant de pieges à sédiment.

Afin d'alléger la suite du texte, le terme phosphore désignera le phosphore total.

\section{DESCRIPTION DU BASSIN VERSANT DE PARELOUP}

\section{II.1 Le bassin versant de Pareloup}

Le bassin versant de la retenue de Pareloup, d'une superficie de $120 \mathrm{~km}^{2}$, est constitué de quatre sous-bassins versants: Vioulou $\left(56,2 \mathrm{~km}^{2}\right)$, Connes $\left(21 \mathrm{~km}^{2}\right)$, Rieutord $\left(15,3 \mathrm{~km}^{2}\right)$ et un bassin versant regroupant un ensemble de petits ruisseaux $\left(26,9 \mathrm{~km}^{2}\right)$. A ces quatre sous-bassins versants s'ajoutent $13 \mathrm{~km}^{2}$ de berges.

L'agriculture, essentiellement orientée vers l'élevage d'ovins et de porcins, occupe près des trois-quarts de la surface du bassin versant. 
La densité de la population sédentaire est faible: elle varie, selon les sous-bassins versants, de 15 à

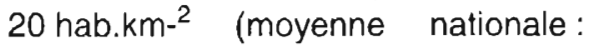
100 hab. $\mathrm{km}^{2}{ }^{2}$ ). Trois agglomérations, Curan, Salles-Curan et Saint-Martindes-Faux, regroupent la majeure partie de la population. Pendant la période estivale, la population est environ multipliée par dix (résidences secondaires et campings situés en bordure directe du lac).

Les réseaux d'assainissement sont rudimentaires: à l'époque de l'étude, seule la commune de Salles-Curan était pourvue d'une station d'épuration de type lagunage naturel. Les campings possèdent leur propre dispositif de collecte des eaux usées.

\section{II.2 La retenue de Pareloup}

Les caractéristiques principales de la retenue et des bassins versants drainés sont résumées dans le tableau I et illustrées par la figure 1 .

\section{BILAN D'EAU}

Le bilan de l'eau entre les entrées et les sorties d'eau détermine la variation journalière de la réserve d'eau dans le lac.

\section{Les entrées regroupent:}

- les trois principaux ruisseaux qui drainent leurs bassins versants respectifs: le Vioulou, le Connes, le Rieutord,
Tableau I. - Caractéristiques principales de la retenue et du bassin versant de Pareloup.

Table I. - Principal characteristics of the Pareloup reservoir and watershed.

\begin{tabular}{|c|}
\hline LAC \\
\hline $\begin{array}{l}\text { Altitude moyenne du plan d'eau : } 800 \mathrm{~m} \\
\text { Superficie: } 1260 \text { ha à la cote } 805 \mathrm{NGF} \\
\text { Volume : } 169 \mathrm{hm}^{3} \text { à la cote } 805 \mathrm{NGF} \\
\text { Profondeur maximale : } 40 \mathrm{~m} \\
\text { Profondeur moyenne : } 12,5 \mathrm{~m} \\
\text { Cote maximale d'exploitation : } 805 \mathrm{~m} \\
\text { Cote minimale d'exploitation : } 775 \mathrm{~m} \\
\text { Débil moyen à l'émissaire : } 1031 . \mathrm{s}^{-1} \\
\text { Temps de séjour moyen : } 300 \mathrm{j} \\
\text { Supericie des berges : } 13 \mathrm{~km}^{2} \\
\text { Débit moyen pompé à Bage : } 3,31 \mathrm{~m}^{3} \cdot \mathrm{s}^{-1} \\
\text { Débit moyen turbiné à Alrance }: 4,94 \mathrm{~m}^{3} \cdot \mathrm{s}^{-1}\end{array}$ \\
\hline BASSIN VERSANT \\
\hline Superficie \\
\hline $\begin{array}{l}\text { Rieutord: } 15,3 \mathrm{~km}^{2} \\
\text { Connes: } 21 \mathrm{~km}^{2} \\
\text { Vioulou : } 56,2 \mathrm{~km}^{2} \\
\text { Pareloup: } 26,9 \mathrm{~km}^{2} \\
\text { Berges: } 13 \mathrm{~km}^{2} \\
\text { supenticie totale: } 122 \mathrm{~km}^{2}\end{array}$ \\
\hline Débits moyens \\
\hline $\begin{array}{l}\text { Rieutord: } 0,21 \mathrm{~m}^{3} \cdot \mathrm{s}^{-1} \\
\text { Connes : } 0,21 \mathrm{~m}^{3} \cdot \mathrm{s}^{-1} \\
\text { Vioulou: } 0,94 \mathrm{~m}^{3} \cdot \mathrm{s}^{-1} \\
\text { Débit réservè du Vioulou: } 0,10 \mathrm{~m}^{3} \cdot \mathrm{s}^{-1}\end{array}$ \\
\hline
\end{tabular}

Altitudes moyennes

Rieutord: $920 \mathrm{~m}$

Connes : $952 \mathrm{~m}$

Vioulou : $984 \mathrm{~m}$

Occupation des bassins versants

Rieutord : terres cultivees : $75 \%$ prairies naturelles + landes : $18,5 \%$ surtaces boisées : $6,5 \%$

Connes : terres cultivées : $70 \%$ prairies naturelles + landes : $21 \%$ surfaces boisees : $9 \%$

Vioulou: terres cultivees : $56 \%$ prairies naturelles + landes : $31 \%$ surfaces boisées : $13 \%$

Rieutord : 20 hab. $\mathrm{km}^{-2}$

Connes: $20 \mathrm{hab}^{\mathrm{km}} \mathrm{.}^{-2}$

Vioulou: 15 hab. $\mathrm{km}^{-2}$ 


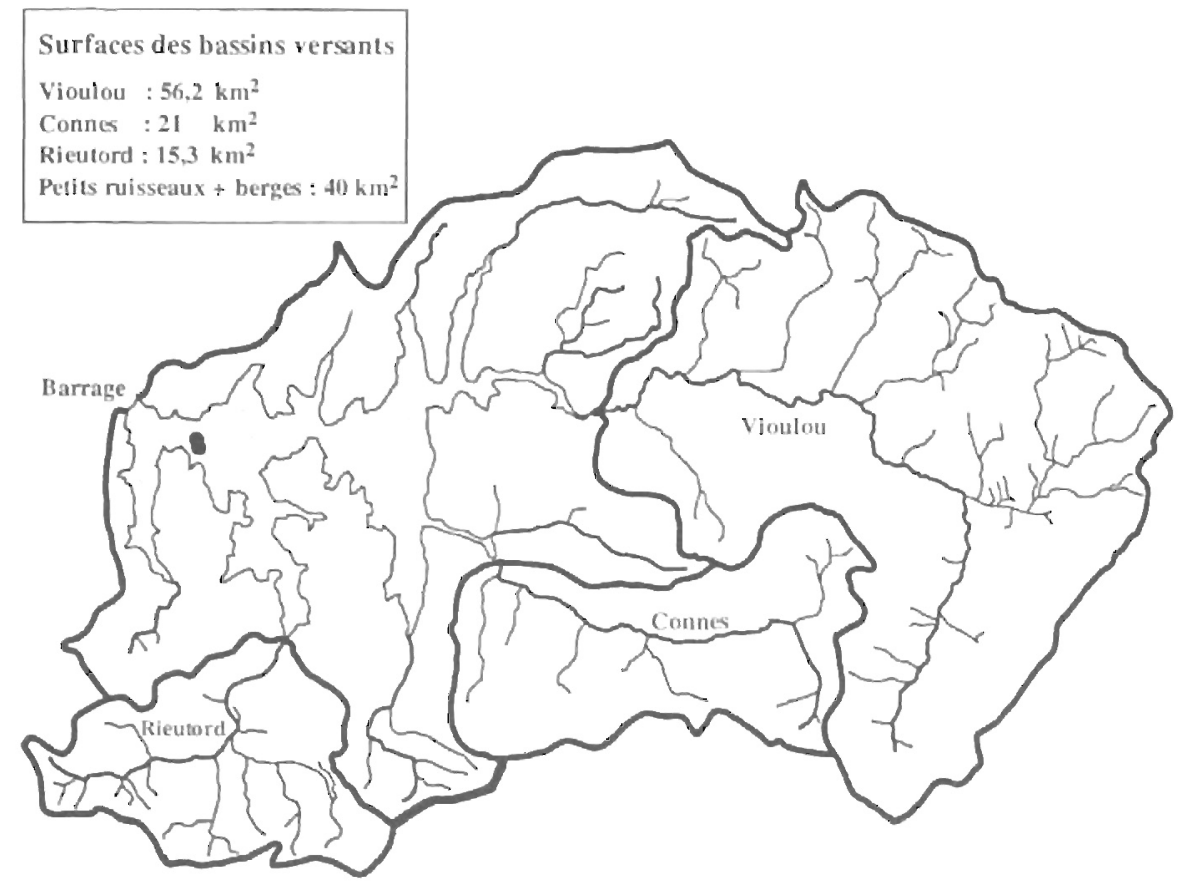

Fig. 1. - Le bassin versant de Pareloup (ocalisation des pieges à sédiment).

Fig. 1. - The Pareloup watershed location of sediment traps).

- les petits ruisseaux du sousbassin versant de Pareloup, du lac,

- les précipitations à la surface

- le ruissellement sur les berges,

- l'eau pompée dans la retenue de Bage.

Les sorties comprennent:

- le débit réservé du Vioulou,

- l'eau turbinée à l'usine d'Alrance, lac.
Le bilan d'eau est calculé quotidiennement du $1^{\text {er }}$ janvier 1986 au 31 décembre 1987 à partir des mesures des débits entrants et sortants.

Pour vérifier l'exactitude du bilan d'eau à l'échelle journalière, les variations calculées de la réserve d'eau du lac sont comparées aux variations observées, obtenues à partir des relevés de cote de la retenue; Iorsque les valeurs observées sont différentes des valeurs calculées, il est nécessaire de compenser cet écart par un bilan de bouclage afin d'obtenir un bilan d'eau équilibré. 


\section{III.1 Traitements des données disponibles}

\section{III.1.1 Les entrées}

Nous disposons des débits moyens journaliers du Vioulou pour toute la période d'étude, mais seulement d'une mesure hebdomadaire de débit pour le Connes et le Rieutord entre le 5 juillet 1986 et le 28 août 1987; pour cette période, les débits moyens quotidiens sont reconstitués par interpolation linéaire entre deux débits mesurés. En dehors de ces dates, et pour couvrir la durée de l'étude, les débits moyens journaliers de ces deux affluents sont calculés à partir des débits journaliers observés du Vioulou et proportionnellement à la superficie de leurs bassins versants respectifs.

En l'absence de données, ce calcul est également appliqué à la détermination des débits moyens journaliers de l'ensemble des petits ruisseaux formant le sous-bassin versant de Pareloup.

Ces estimations sont basées sur l'hypothèse que ces bassins voisins sont suffisamment proches pour que les précipitations et l'état des sols soient similaires. Alors, le débit dans le Vioulou est un bon témoin du régime hydrologique de ces bassins, moyennant une correction de superficie.

La valeur des apports directs par précipitation est calculée à partir des relevés quotidiens de hauteur de pluie et de la surface du plan d'eau.

Les apports directs par ruissellement sont calculés à partir des préci- pitations sur la surface des berges, les pertes par infiltration n'étant pas prises en compte.

Enfin, à ces entrées, s'ajoutent les débits moyens journaliers pompés à Bage.

\section{III.1.2 Les sorties}

Nous disposons de mesures quotidiennes du débit réservé du Vioulou à l'aval du barrage et du débit turbiné à Alrance.

Le calcul de l'évaporation à la surface du plan d'eau est présenté dans le tableau II.

\section{III.1.3 Les variations de réserve d'eau dans la retenue}

La gestion de la retenue étant de type saisonnier, le volume emmagasiné dans le plan d'eau permet d'optimiser la production hydroélectrique. Cette gestion correspond:

- à un stockage des apports pendant les périodes de moindre besoin en électricité,

- au maintien d'une cote haute et stable en été pendant la période touristique,

- au turbinage en hiver pendant les périodes de forte demande en électricité.

Les relevés quotidiens de cote du lac et une courbe de capacité (fig. 2) permettent de calculer les variations journalières de volume et de surface.

Le tableau III résume la fréquence des mesures et les traitements appliqués à ces données pour reconstituer 
Tableau II. - Calcul du débit d'évaporation à la surface d'un plan d'eau (d'après Salençon et al., 1976).

Table II. - Calculation of the rate of evaporation at the surface of a reservoir (after Salençon et al., 1976).

Le débit d'évaporation à la surface d'un plan d'eau est calculé par l'équation (Salençon et al., 1976) :

$$
D=\rho_{a} C(u)\left(Q_{\theta}-Q_{a}\right) \underset{\rho_{e}}{S}
$$

$D$ : débit d'eau évaporée $\left(m^{3} \cdot s^{-1}\right)$

$\rho_{a}:$ masse volumique de l'air $=1,293 \mathrm{~kg} \cdot \mathrm{m}^{-3}$

$\rho_{e}:$ masse volumique de l'eau $=1000 \mathrm{~kg} \cdot \mathrm{m}^{\cdot 3}$

$\mathrm{Qe}$ : humidité spécifique de l'air saturé de la couche limite au-dessus du plan d'eau ( $\left.\mathrm{kg}^{\mathrm{kg}}{ }^{-1}\right)$

$\mathrm{Qa}:$ humidité spécifique de l'air $\left(\mathrm{kg}^{\mathrm{kg}}{ }^{-1}\right)$

$\mathrm{C}(\mathrm{u})$ : fonction de transfert empirique entre la station météorologique et les conditions météorologiques locales

S : surface du plan d'eau $\left(\mathrm{m}^{2}\right)$

Calcul de $C(u): C(u)=a(1+u)$

a (sans dimension) : $0,0015<\mathrm{a}<0,0025$

$\mathrm{u}$ : vitesse du vent à $2 \mathrm{~m}$ d'altitude $\left(\mathrm{m} \cdot \mathrm{s}^{-1}\right)$

Calcul de l'humidité spécifique de l'air saturé de la couche limite au dessus du plan d'eau Qe $\left(\mathrm{kg} \cdot \mathrm{kg}^{-1}\right)$ :

$$
\text { Qe. } \begin{gathered}
0,622 E O \\
\mathrm{~Pa}-0,378 \mathrm{EO}
\end{gathered}
$$

$\mathrm{Pa}$ : pression atmosphérique $(\mathrm{hPa})$

EO : pression de vapeur saturante $(\mathrm{hPa})$ à la température de l'eau ( $\mathrm{Te}$ en ${ }^{\circ} \mathrm{C}$ ) calculée à partir de la formule de Magnus-Tetens :

$$
E O=\exp \left(2,3026\left(\begin{array}{c}
7,5 T e \\
T e+273,3
\end{array}+0,7858\right)\right)
$$

Calcul de l'humidité spécifique de l'air Qa $\left(\mathrm{kg}^{\mathrm{kg}} \mathrm{kg}^{-1}\right)$ :

$$
\mathrm{Qa}-\begin{gathered}
0,622 \mathrm{EA} \\
\mathrm{Pa}-0,378 \mathrm{EA}
\end{gathered}
$$

EA : pression partielle de la vapeur d'eau dans l'air ( $\mathrm{hPa}$ )

$$
E A=\stackrel{H}{100} E W A
$$

$\mathrm{H}$ : humidité relative de l'air (\%)

EWA : pression de vapeur saturante $(\mathrm{hPa})$ à la température de l'air $\left(\mathrm{Ta}\right.$ en ${ }^{\circ} \mathrm{C}$ ) calculée à partir de la formule de Magnus-Tetens:

$$
E W A=\exp \left(2,3026\left(\begin{array}{c}
7,5 T a \\
T a-273,3
\end{array}-0,7858\right)\right)
$$




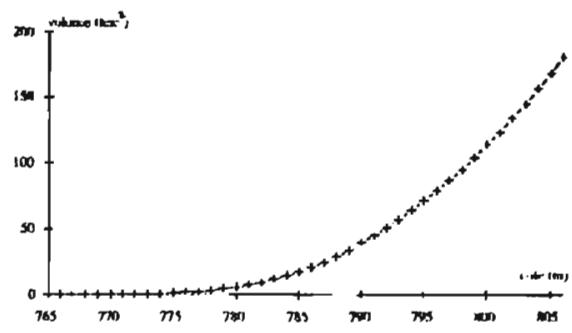

Fig. 2. - Courbe de capacité de la retenue de Pareloup.

Fig. 2. - Capacity curve for Pareloup reservoir. les débits journaliers de l'ensemble des entrées et sorties.

\section{III.2 Résultats et répartition du bilan de bouclage}

Le bilan en eau (fig. 3) met bien en évidence la fonction de réservoir de stockage de la retenue de Pareloup. En moyenne, plus de la moitié de l'eau entrant dans la retenue est de

Tableau III. - Fréquence des mesures et traitements appliqués à ces données pour reconstituer les débits journaliers de l'ensemble des entrées et sorties.

Table III. - Frequency of readings and processing of data to reconstitute daily flow rates for all inflow and outflow.

\begin{tabular}{|c|c|c|}
\hline \multirow{3}{*}{ Vioulou } & \multicolumn{2}{|c|}{ BILAN EN EAU } \\
\hline & Fréquence des données & Traitement des données \\
\hline & débits moyens journaliers $\left(\mathrm{m}^{3} \cdot \mathrm{s}^{-1}\right)$ & débits moyens journaliers $\left(\mathrm{m}^{3} \cdot \mathrm{j}^{-1}\right)$ \\
\hline $\begin{array}{l}\text { Rieutord } \\
\text { Connes }\end{array}$ & $\begin{array}{l}\text { débits moyens journaliers }\left(\mathrm{m}^{3} \cdot \mathrm{s}^{-1}\right) \\
\text { tous les } 7 \text { jours du } 5 / 7 / 86 \text { au } \\
28 / 8 / 87 \\
\end{array}$ & $\begin{array}{l}\text { débits moyens journaliers }\left(\mathrm{m}^{3} \cdot \mathrm{j}^{-1}\right) \\
\text { - interpolation linéaire entre deux } \\
\text { débits mesurés } \\
\text { - interpolation à partir des débits } \\
\text { mesurès du Vioulou et proportion- } \\
\text { nellement à la superficie des bas- } \\
\text { sins versants respectifs }\end{array}$ \\
\hline Pareloup & pas de données & $\begin{array}{l}\text { - interpolation à partir des débits } \\
\text { mesurés du Vioulou et proportion- } \\
\text { nellement à la superficie des bas- } \\
\text { sins versants respectifs }\end{array}$ \\
\hline Bage & débits moyens journaliers $\left(\mathrm{m}^{3} \cdot \mathrm{s}^{-1}\right)$ & débits moyens journaliers $\left(\mathrm{m}^{3} \cdot \mathrm{j}^{-1}\right)$ \\
\hline Précipitations & hauteur d'eau quotidienne (mm) & $\begin{array}{l}\text { produit de la hauteur d'eau et de } \\
\text { la surface du lac au jour considéré } \\
\left(\mathrm{m}^{3}\right)\end{array}$ \\
\hline Ruissellement & pas de données & $\begin{array}{l}\text { produit de la hauteur d'eau et de } \\
\text { la surface des berges au jour } \\
\text { considéré }\left(\mathrm{m}^{3}\right)\end{array}$ \\
\hline Alrance & débits moyens journaliers $\left(\mathrm{m}^{3} \cdot \mathrm{s}^{-1}\right)$ & đébits moyens journaliers $\left(\mathrm{m}^{3} \cdot \mathrm{j}^{-1}\right)$ \\
\hline $\begin{array}{l}\text { débit réservé } \\
\text { Vioulou }\end{array}$ & débits moyens journaliers $\left(\mathrm{m}^{3} \cdot \mathrm{s}^{-1}\right)$ & débits moyens journaliers $\left(\mathrm{m}^{3} \cdot \mathrm{j}^{-1}\right)$ \\
\hline Evaporation & I volumes quotidiens évaporés $\left(\mathrm{m}^{3} \cdot \mathrm{s}^{-1}\right.$ & ) volumes évaporés $\left(\mathrm{m}^{3} \cdot j^{-1}\right)$ \\
\hline
\end{tabular}




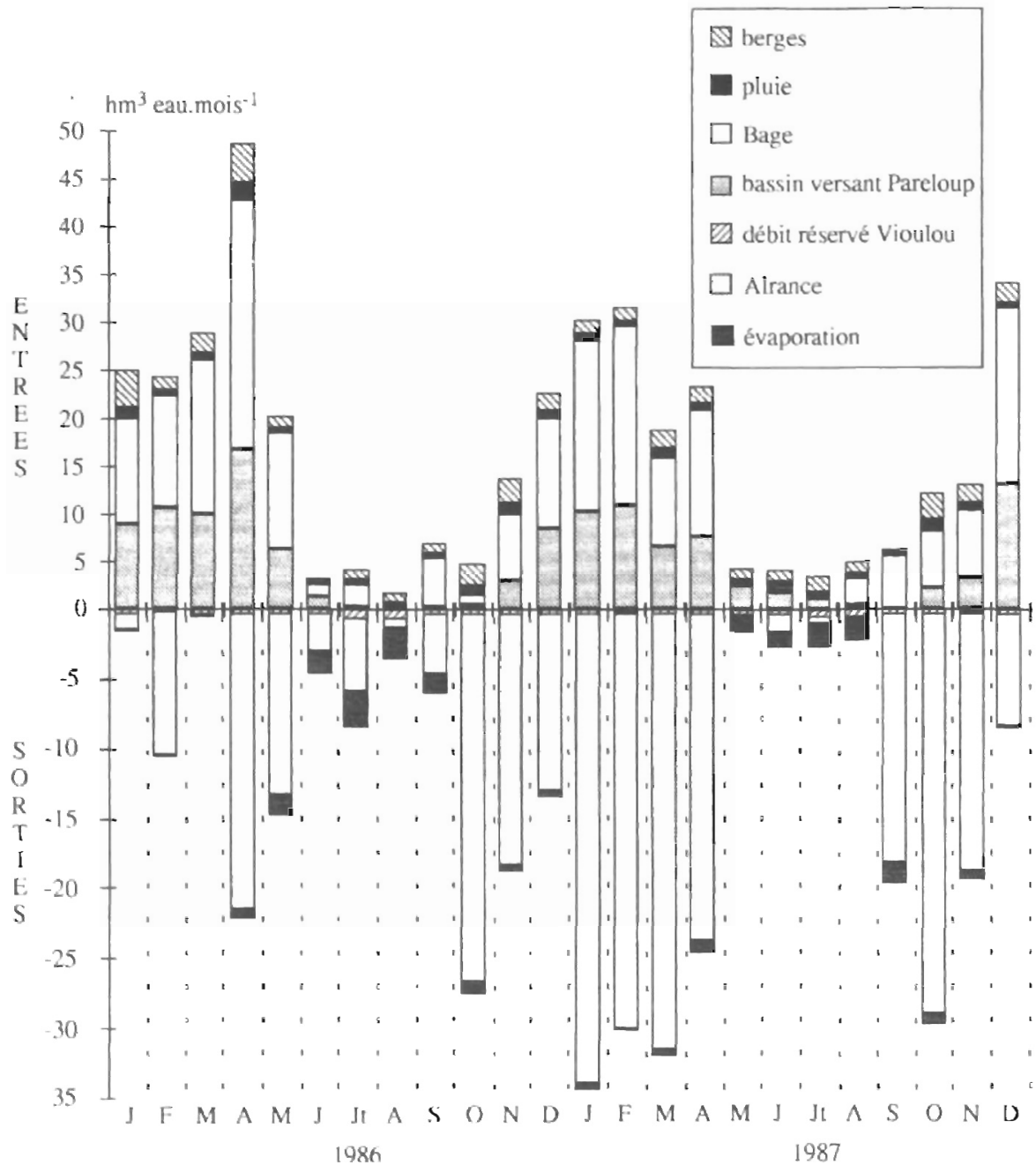

Fig. 3. - Le bilan d'eau: importance relative des différentes entrées et sorties.

Fig. 3. - Annual water budget: relative importance of the various inputs and outputs.

l'eau pompée dans la retenue de Bage $(52 \%$ en 1986 et $54 \%$ en 1987); le bassin versant n'apporte que $32 \%$ de l'eau. Les sorties d'eau sont essentiellement constituées par l'eau turbinée à Alrance $190 \%$ en moyenne).
Pour les deux années d'étude, la comparaison entre les variations mensuelles de volume de la retenue, déduites des relevés de cote, et les variations de volume calculées à partir des débits entrants et sortants (fig. 4) fait apparaître une sous-estimation 


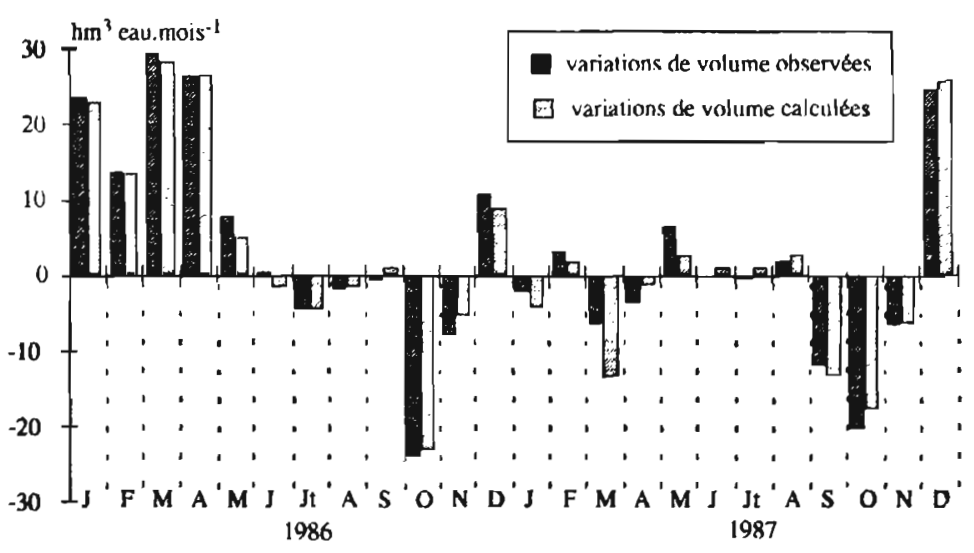

Fig. 4. - Comparaison entre les variations mensuelles de volume observées (déduites des relevés de cote de la retenue) et les variations de volume calculées à partir des débits entrants et sortants. Fig. 4. - Comparison between observed monthly variations in volume (deduced from reservoir level readings) and variations in volume calculated from inflow and outflow.

des apports : $-3,04 \mathrm{hm}^{3}$ d'eau (soit $2,41 \%$ du volume moyen annuel du lac) pour 1986 et $-4,91 \mathrm{hm}^{3}$ d'eau (soit 4,21\%) pour 1987.

Cette sous-estimation peut avoir plusieurs causes:

- les différentes méthodes d'intégration de la courbe de capacité peuvent conduire à des estimations de volume différentes du volume réellement stocké;

- pour une superficie de $12 \mathrm{~km}^{2}$, une erreur de $1 \mathrm{~cm}$ sur la lecture de la cote du plan d'eau correspond à un débit moyen journalier de $1,5 \mathrm{~m}^{3} \cdot \mathrm{s}^{-1}$ $\left(0,13 \mathrm{hm}^{3} \cdot \mathrm{j}^{-1}\right)$. Si les difficultés de lecture de l'échelle sont augmentées par les vagues, il ne faut pas non plus négliger les erreurs induites par l'inclinaison de la surface libre sous l'effet du vent (seiches-upwelling) (Salençon et Calmels, 1994);
- la reconstitution des débits manquants par interpolation linéaire ou par référence aux débits du Vioulou sont des sources d'erreur. Pour les quelques dates où nous disposons des débits réels des trois affluents, nous avons comparé ces derniers aux débits calculés. La figure 5 montre, qu'au moins pour les faibles débits, le calcul au prorata des superficies des bassins versants sous-estime les débits du Connes et du Rieutord. Ceci est lié au fait que le débit mesuré du Vioulou n'est pas représentatif de la superficie de son bassin versant mais d'un bassin versant d'une superficie moindre, certaines sources situées en amont étant captées par la ville de Rodez pour son alimentation en eau potable. Si ce captage n'a qu'une incidence négligeable sur le débit du Vioulou en période de hautes eaux, il n'en est pas de même en période de faibles débits. 


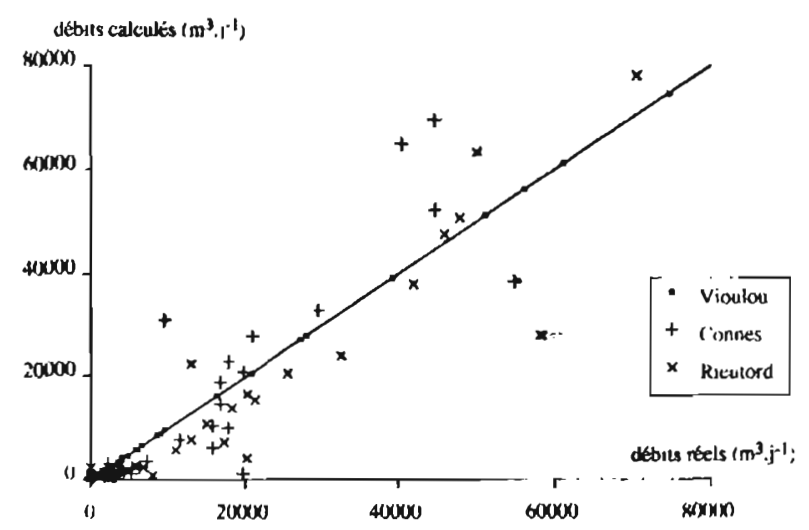

Fig. 5. - Comparaison entre les débits réels du Connes et du Rieutord et leurs débits estimés à partir de ceux du vioulou.

Fig. 5. - Comparison between measured flow rates of the Connes and Rieutord and the respective rates calculated from those of the Vioulou.

Les deux dernières causes du déficit en eau nous ont conduits à répartir ce déficit dans le bilan de bouclage pour $1 / 4$ sur le volume du lac et pour $3 / 4$ sur le débit du Rieutord. Nous avons préféré le Rieutord au Connes car ce dernier draine des concentrations élevées de phosphore provenant de la station d'épuration de Salles-Curan et ne serait pas représentatif pour le bilan de phosphore.

La répartition du déficit en eau sur le volume du lac a volontairement été limitée à $1 / 4$, une proportion plus élevée signifiant une erreur improbable de lecture de la cote.

\section{BILAN DU PHOSPHORE}

Les variations quotidiennes du stock de phosphore de la retenue résultent du bilan entre les entrées et sorties de phosphore. Les entrées et les sorties sont les mêmes que pour le bilan d'eau exceptée l'évaporation.

La charge en phosphore est le produit entre le débit et la concentration de chaque entrée ou sortie.

\section{IV.1 Traitement des données disponibles}

Nous avons calculé le bilan en phosphore du $1^{\text {er }}$ avril 1986 au 31 juillet 1987, seule période pour laquelle nous disposions des données pour l'ensemble du bassin versant.

\section{IV.1.1 Les entrées}

Le bassin versant de Pareloup étant essentiellement rural, les apports de phosphore ont surtout une origine diffuse (lessivage des sols agricoles); les apports ponctuels sont limités à la pollution domestique, principalement 
en période touristique et aux apports de Bage.

La concentration en phosphore des trois principaux affluents (Vioulou, Connes, Rieutord) est mesurée à partir de prélèvements hebdomadaires réalisés à l'exutoire des bassins versants. Entre deux mesures, les concentrations sont estimées par interpolation linéaire.

Neuf épisodes de crues et de hautes eaux ont été suivis à intervalles réguliers sur le Vioulou. Nous disposons des mesures de débit et de concentration en phosphore, toutes les deux heures pour les crues et toutes les quatre heures pour les hautes eaux. Elles nous ont permis de déduire les débits et concentrations moyennes journalières.

Les apports des petits ruisseaux de Pareloup sont évalués à partir des apports mesurés du Vioulou proportionnellement à la superficie des sous-bassins versants.

L'apport de phosphore par les précipitations n'a pas été pris en compte en raison de l'absence de mesures sur le site. Selon Rast et Lee (1978), cet apport ne représenterait que $0,02 \mathrm{~g} \cdot \mathrm{m}^{-2} \cdot \mathrm{an}^{-1}$ soit $0,25 \mathrm{~kg} \cdot \mathrm{an}^{-1}$ pour la surface moyenne du plan d'eau.

Les apports par ruissellement sur les berges sont évalués à partir des charges moyennes annuelles des affluents. Le coefficient d'exportation ainsi calculé est de $0,25 \mathrm{~kg} \cdot \mathrm{ha}^{-1} \cdot \mathrm{an}^{-1}$.

La quantité de phosphore rejetée par la pollution domestique a été estimée à partir de données bibliographiques $\left(4 \mathrm{~g} \cdot \mathrm{hab}^{-1} \cdot \mathrm{j}^{-1}\right)$. Ces apports imputables à la population permanente sont multipliés par dix pendant les deux mois de la période estivale.

\section{IV.1.2 Cas particulier de Bage}

Les concentrations journalières en phosphore de l'eau pompée à Bage sont reconstituées par interpolation linéaire à partir de mesures hebdomadaires.

Par rapport aux années précédant cette étude, 1986 et 1987 sont caractérisées par une forte augmentation des concentrations de phosphore dans l'eau pompée à Bage (200 à $250 \mu \mathrm{g} . \mathrm{I}^{-1}$ contre $50 \mu \mathrm{g} . \mathrm{I}^{-1}$ en moyenne avant 1986). Les matières en suspension (MES) ont augmenté dans les mêmes proportions. Cette augmentation est également constatée dans les affluents, mais elle est bien moindre. Son origine n'est pas clairement définie, peut-être est-elle due à des opérations de drainage sur les terres du bassin versant de Pont-de-Salars.

Cependant, outre l'augmentation moyenne du phosphore dans les eaux de Bage, on observe durant l'hiver 8687 un pic atteignant $2500 \mu \mathrm{g} \cdot \mathrm{l}^{-1}$ pendant deux mois. Ce gros apport de phosphore est dû à un rejet accidentel d'une charcuterie industrielle qui a provoqué une forte eutrophisation de la retenue de Pont-de-Salars et une pollution importante du Viaur ayant entraîné la mort de nombreuses truites. Toutefois, ce phosphore n'est retrouvé ni dans le lac au point de prélèvement, ni dans le Vioulou aval, ni dans les pièges à sédiment. 
Nous ne disposons pas des mesures de concentration en phosphore de l'eau turbinée à Alrance. Aussi, afin de déterminer si le phosphore pouvait être évacué dans l'eau turbinée, compte tenu de la proximité de la conduite de Bage et de la prise d'eau d'Alrance, nous avons comparé les variations de concentration de phosphore dans les couches profondes de la retenue aux différentes séquences de pompage-turbinage. Si le pompage n'est pas suivi d'un turbinage, on observe un pic de phosphore à $-25 \mathrm{~m}$ (par exemple 10 juin, 5 août, $1^{\text {er }}$ septembre 1987); au contraire si pompage et turbinage sont consécutifs, les variations de concentration dans les couches profondes sont faibles (par exemple 3 juin 1986, 29 avril $1987, \ldots)$. Cependant cette relation n'est pas systématique, en particulier durant l'hiver 1986-1987.

D'autre part, les campagnes de traçage ont montré qu'en période stratifiée, l'eau turbinée immédiatement après un pompage de fin de semaine ne contenait que $6 \%$ d'eau de Bage (Calmels et Salençon, 1991; Salençon et Calmels, 1994).

Aussi est-il probable que le phosphore sédimente rapidement près de la conduite d'amenée. Le faible débit réservé explique que I'on n'en trouve pas trace dans le Vioulou aval.

Ceci nous a conduits à ramener les valeurs extrêmes relevées pendant l'hiver 86-87 aux valeurs les plus hautes observées durant le reste de la période d'étude $\left(250 \mu \mathrm{g} . \mathrm{I}^{-1}\right)$.

\section{IV.1.3 Les sorties}

Nous avons considéré que l'eau turbinée avait une concentration en phosphore égale à la concentration moyenne dans l'hypolimnion en période de stratification et égale à celle de la colonne d'eau en période d'isothermie.

Les concentrations journalières du débit réservé du Vioulou sont reconstituées par interpolation linéaire à partir de mesures hebdomadaires.

Le tableau IV présente, pour chaque élément d'entrée et de sortie, les fréquences des mesures et les traitements appliqués à ces données pour reconstituer les concentrations journalières.

\section{IV.1.4 Les variations de stock de phosphore de la retenue}

Les concentrations en phosphore de la retenue sont mesurées toutes les semaines, à différentes profondeurs sur une verticale. Les variations journalières de stock de phosphore de la retenue sont calculées à partir des concentrations moyennes journalières reconstituées par interpolation linéaire.

\section{IV.2 Résultats}

Le phosphore apporté dans la retenue pendant les 16 mois étudiés est estimé à 29 t. Cet apport fluctue au cours de l'année (fig. 6a). Le maximum est atteint en hiver (décembre à février). En juillet et août, les apports en phosphore sont importants $\left(2,1\right.$ t.mois $\left.^{-1}\right)$. 
Tableau IV. - Fréquence des mesures et traitements appliqués à ces données pour reconstituer les concentrations journalières de phosphore de l'ensemble des entrées et sorties.

Table IV. - Frequency of readings and processing of data to reconstitute daily concentrations of phosphorus for all inflow and outflow.

\begin{tabular}{|c|c|c|c|}
\hline & & BILAN & EN PHOSPHORE \\
\hline & & Fréquence des données & Traitement des données \\
\hline & $\begin{array}{l}\text { Vioulou } \\
\text { Rieutord } \\
\text { Connes }\end{array}$ & $\begin{array}{l}\text { mesure hebdomadaire de } \\
\text { concentration } \\
\left(\mathrm{mg} \mathrm{P.m} \mathrm{m}^{-3}\right)\end{array}$ & $\begin{array}{l}\text { interpolation linéaire entre deux } \\
\text { concentrations mesurées } \\
\left.1 \text { (mg P.m }{ }^{-3}\right)\end{array}$ \\
\hline E & Pareloup & pas de données & $\begin{array}{l}\text { interpolation proportionnellement à la } \\
\text { charge du Vioulou }\end{array}$ \\
\hline Ŕ & Bage & $\begin{array}{l}\text { mesure hebdomadaire de } \\
\text { concentration }\left(\mathrm{mg} \mathrm{P} \cdot \mathrm{m}^{-3}\right)\end{array}$ & $\begin{array}{l}\text { interpolation linéaire entre deux } \\
\text { concentrations mesurées }\end{array}$ \\
\hline $\begin{array}{l}E \\
S\end{array}$ & Précipitations & pas de données & ' négligées \\
\hline & Ruissellement & pas de données & coefficient d'export $\left(=0,25 \mathrm{~kg} \cdot \mathrm{ha}^{-1} \cdot \mathrm{an}^{-1}\right)$ \\
\hline & Apports domestiques & I pas de données & coefficient d'export $\left(=4 \mathrm{~g} \cdot \mathrm{hab}^{-1} \cdot \mathrm{j}^{-1}\right)$ \\
\hline $\begin{array}{l}S \\
O \\
R \\
T\end{array}$ & Alrance & $\begin{array}{l}\text { mesure hebdomadaire de } \\
\text { concentration de la retenue } \\
\left(\mathrm{mg} \mathrm{P.m} \mathrm{m}^{-3} \text { ) }\right.\end{array}$ & $\begin{array}{l}\text { interpolation linéaire entre les } \\
\text { : moyennes des concentrations } \\
\text { - sous la thermocline (stratification) } \\
\text { - de l'ensemble de la colonne } \\
\text { d'eau (isothermie) }\end{array}$ \\
\hline E & Débit réservé Vioulou & $\begin{array}{l}\text { mesure hebdomadaire de } \\
\text { concentration de la retenue } \\
\left(\mathrm{mg} \mathrm{P.m}^{-3}\right)\end{array}$ & $\begin{array}{l}\text { interpolation linéaire entre doux } \\
\text { concentrations mesurées }\end{array}$ \\
\hline & dimentation & pas de données & $\begin{array}{l}\text { différence entre le bilan entrées-sor- } \\
\text { ties de phosphore et la variation du } \\
\text { stock de phosphore de la retenue }\end{array}$ \\
\hline
\end{tabular}

ils sont essentiellement dus à la pollution domestique saisonnière (en moyenne $93 \%$ des apports en phosphore). Si l'on excepte les apports domestiques, plus des 2/3 du phosphore entrant dans la retenue proviennent des bassins versants de Bage et du Viaur par pompage alors que cet apport d'eau ne représente que $53 \%$ des apports d'eau de la retenue. Ceci confirme que les eaux provenant de ces bassins versants sont plus riches en phosphore que celles du bassin versant de Pareloup.

L'hydrogramme du Vioulou et l'évolution générale du phosphore total (fig. 7a) caractérisent deux types de crues: les unes interviennent après des étiages, les autres en période de hautes eaux. Les figures $7 b$ et $7 c$ illustrent l'évolution horaire des débits, du phosphore total et des orthophos- 


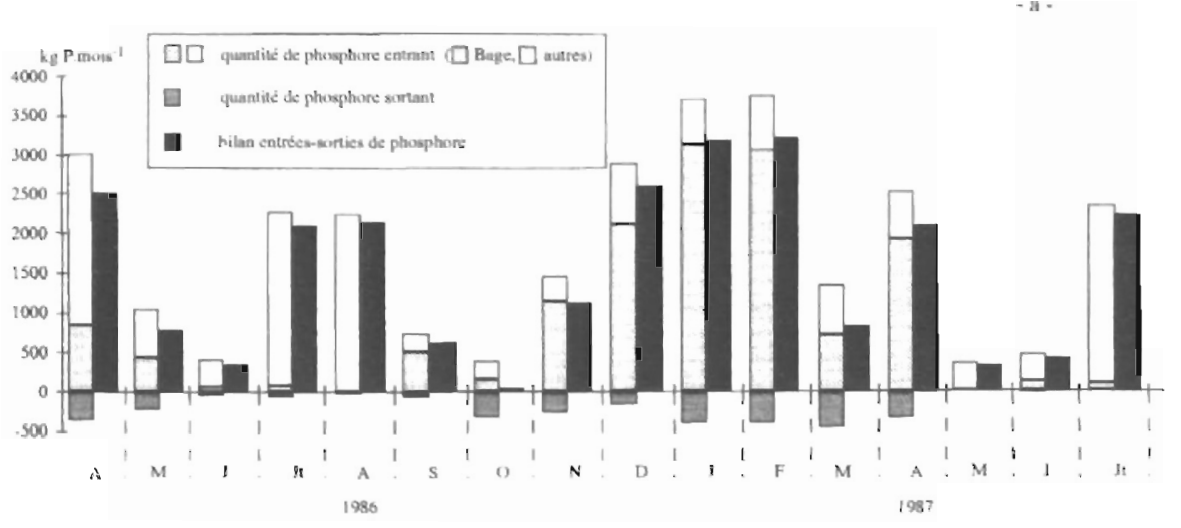

$-\mathrm{b} \cdot$
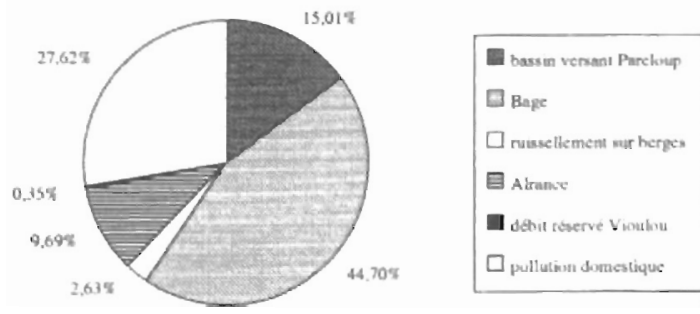

Fig. 6. - Le bilan d'entrées-sortios de phosphore : bilans mensuels (a) et importance relative pour l'ensemble de la période des différentes entrées et sorties de phosphore (b).

Fig. 6, - Phosphorus input-output budget: monthly budgets (a) and relative importance of various inputs and outputs over the entire period (b).

phates pour les deux types de crues; l'évolution de ces trois variables est analogue pour les sept autres épisodes de crues et de hautes eaux qui ont été suivis. Les relations entre les débits, les MES et le phosphore total rassemblent les observations réalisées lors de neuf crues en 1986-1987 et de deux crues en 1985 (12 avril et 8 mai) (fig. 8).

En période de crues, la remobilisation du phosphore est importante et se fait surtout sous forme de phos- phore particulaire; les teneurs en orthophosphates sont constantes quel que soit le débit.

Lors des premières crues après la saison sèche, les concentrations maximales de phosphore total sont enregistrées avant le débit maximum; les matières en suspension ont une teneur élevée en phosphore de l'ordre de $1,85 \mathrm{~g}^{\mathrm{kg}}{ }^{-1}$ MES. Ces crues mobilisent, mêmè pour de faibles débits, une grande quantité de phosphore, phosphore qui s'était progressivement 


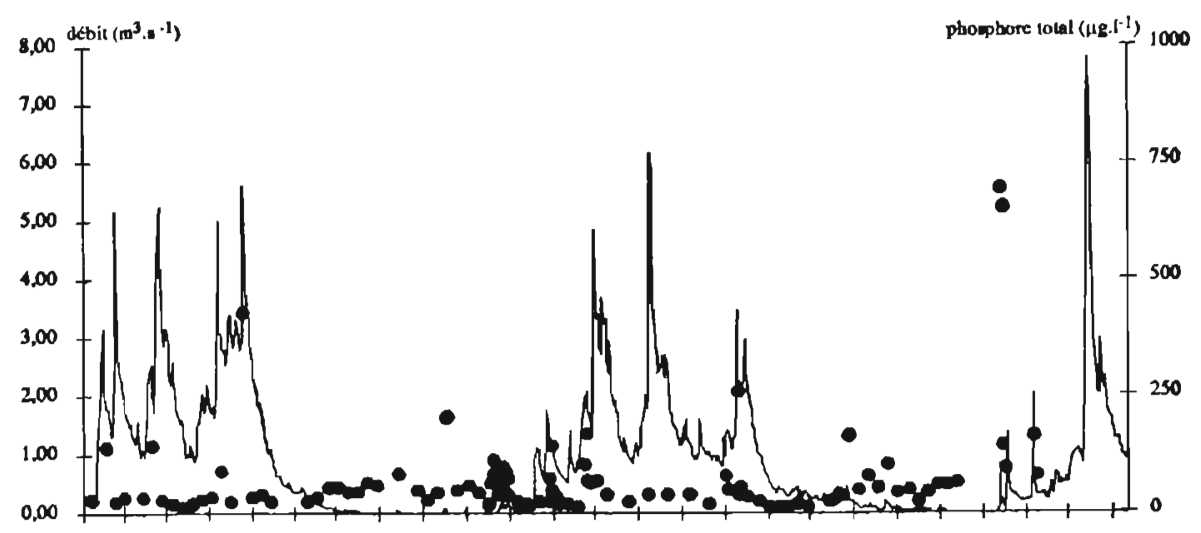

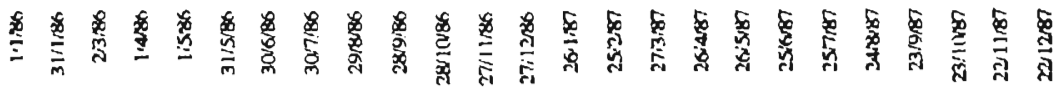
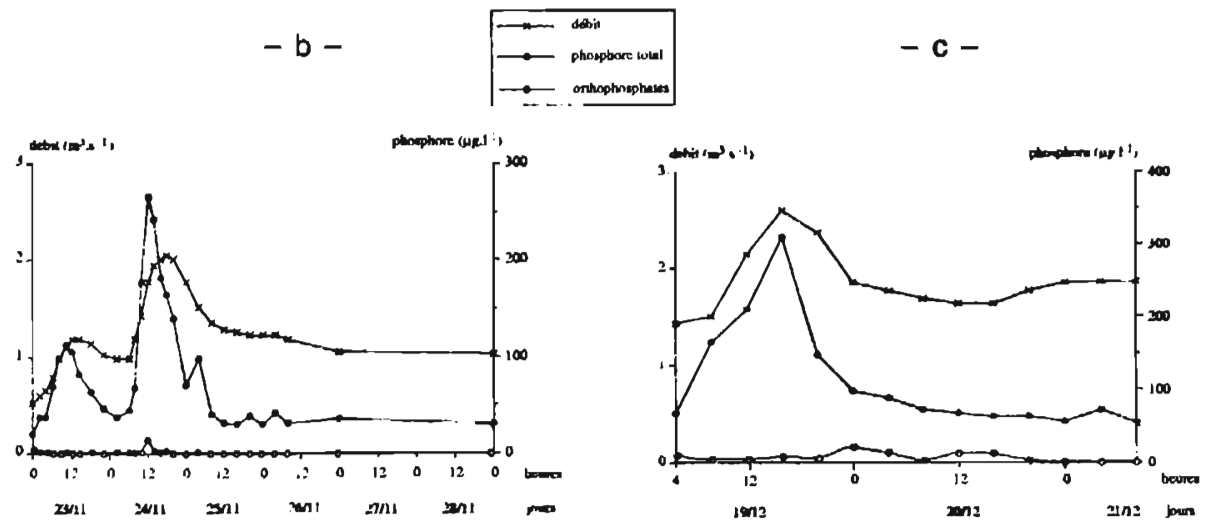

Fig. 7. - Evolution des débits et des concentrations en phosphore total du Vioulou en période de crues et de hautes eaux.

a - Hydrogramme du Vioulou du 1-1-1986 au 31-12-1987 et évolution saisonnière du phosphore total.

b et $\mathrm{c}$ - Evolution des débits, des concentrations en orthophosphates et phosphore total au cours des crues du 24-11-1986 (b) et du 19-12-1986 (c).

Fig. 7. - Evolution of flow rates and total phosphorus concentrations for the Vioulou during spate and high-water periods:

a - Hydrogram of the Vioulou from 1 January 1986 to 31 December 1987, and seasonal variations in total phosphorus

$\mathrm{b}$ and $\mathrm{c}$ - Evolution of flow rates, orthophosphate concentrations and total phosphorus during the spates of 24 November 1986 (b) and 19 December 1986 (c). 

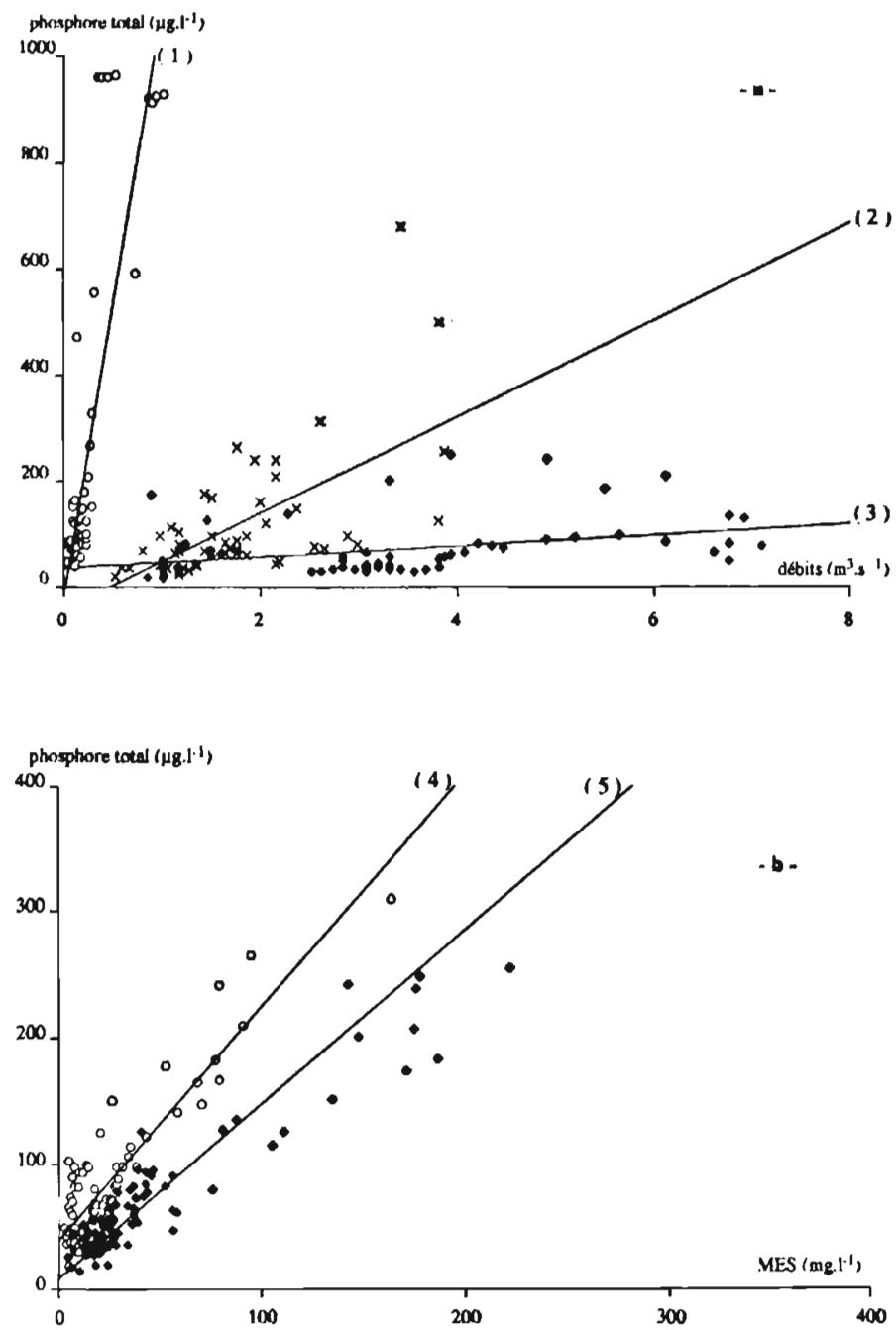

accumulé dans le lit de la rivière en période d'étiage et qui a été entraîné par le front de la crue. Le même phénomène a lieu en période d'étiage où une très faible variation de débit suffit à mobiliser beaucoup de phosphore (13 septembre 1986).
Par contre, en période de hautes eaux, la remobilisation du phosphore est faible ou modérée bien que de forts débits soient atteints. Les concentrations maximales en phosphore sont en général enregistrées à la pointe de crue; la teneur moyenne 
en phosphore des MES est plus faible qu'en période d'étiage, de l'ordre de $1,4 \mathrm{~g} \cdot \mathrm{kg}^{-1}$. En période de hautes eaux, le débit est suffisant pour emporter le phosphore qui n'a plus le temps de s'accumuler. Ces observations sont conformes à celles de Meybeck (1985) et Dorioz et al. (1986).

Les sorties de phosphore sont faibles comparées aux apports et ne représentent que $10 \%$ des entrées, soit un cumul de $3,24 \mathrm{t}$ pour la période d'étude (fig. 6b).

Le bilan entre les entrées et les sorties, c'est-à-dire le stock accumulé dans la retenue, est évalué à $25,75 \mathrm{t}$ entre le $1^{\text {er }}$ avril 1986 et le 31 juillet 1987. La figure $6 b$ résume la part relative de chacune des entrées et sorties de phosphore. Ces résultats corroborent ceux de Bosc (1985).
II existe un certain nombre de modèles simples, établis à partir de régressions sur un grand nombre de lac et réservoirs, permettant d'estimer le niveau trophique d'un réservoir à partir de la charge annuelle en phosphore. Nous en avons testé trois, adaptés aux réservoirs : le modèle de Vollenweider (1976), le modèle de Canfield et Bachmann (1981), le modèle OCDE (1982). Ces modèles utilisent les données globales:

$Z$ : la profondeur moyenne $(12,5 \mathrm{~m})$

$A_{0}$ : la superficie du réservoir $\left(12,6010^{6} \mathrm{~m}^{2}\right)$

$\tau_{w}$ : le temps de séjour (300 jours $=0,82$ an)

$L$ : la charge annuelle en phosphore (en mg.an ${ }^{-1}$ )

$P_{1}$ : la concentration moyenne annuelle en phosphore dans le lac ( $\mu \mathrm{g}$ P. ${ }^{-1}$ )

Fig. 8. - Relation entre le phosphore, les MES et les débits

a - Evolution du phosphore en fonction des débits. Trois nuages de points peuvent être individualisés:

(1) - crues fortement mobilisatrices ayant lieu après des étiages prononcés (14-10-1986, 21 10-1986, 28-10-1987);

(2) - crues modérément mobilisatrices (23-11-1986, 18-12-1986, 3-4-1987);

(3) - crues faiblement mobilisatrices ayant lieu en période de hautes eaux (11-4-1985, 7-5-1985, 26-3-1987)

b - Relation entre le phosphore et les MES. Deux nuages de points peuvent être individualisés :

(4) - fortes teneurs en phosphore des MES en période d'étiage : $1,85 \mathrm{~g}_{\mathrm{kg}}{ }^{-1} \mathrm{MES} \pm 0,2$;

(5) - faibles teneurs en phosphore des MES en période de hautes eaux: $1,38 \mathrm{~g} \cdot \mathrm{kg}^{-1} \mathrm{MES} \pm$ 0,1 .

Fig. 8. - Relationship between phosphorus, suspended solids (MES) and flow rate:

a - Evolution of phosphorus as a function of flow. Three clouds of points can be distinguished:

(1) highly mobilizing spates following pronounced low-water periods (14 Oct. 86, 21 Oct. 86, 28 Oct. 87)

(2) moderately mobilizing spates (23 Nov. 86, 18 Dec. 86, 3 April 87)

(3) little mobilizing spates during high-water periods (11 April 85, 7 May 85, 26 March 87)

b - Relationship belween phosphorus and MES. Two clouds of points can be distinguished:

(4) high phosphorus concentrations in suspended solids during low-water periods: $1.85 \mathrm{~g}^{\mathrm{kg}} \mathrm{kg}^{-1} \pm 0.2$

(5) low phosphorus concentrations in suspended solids during high-water periods: $1.38 \mathrm{~g} \cdot \mathrm{kg}^{-1} \pm 0.1$. 
L'équation de Vollenweider (1976) permet de déterminer si la charge en phosphore constitue un risque d'eutrophisation. Les concentrations "tolérables" et "dangereuses" d'un lac

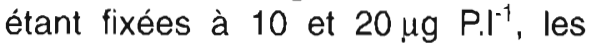
charges "tolérable", $L_{T}(P)$, et "dangereuse", $L_{D}(P)$ sont respectivement égales à:

$$
\begin{aligned}
& L_{T}(P)=A_{0}\left(100+\frac{10 Z}{\tau_{w}}\right) \\
& L_{D}(P)=A_{0}\left(100+\frac{20 Z}{\tau_{w}}\right)
\end{aligned}
$$

A Pareloup, la charge "tolérable" serait de $3,13 \mathrm{t}^{-a^{-1}}$ et la charge "dangereuse" de 6,25 t.an"-1. Pour le cycle annuel du $1^{\text {er }}$ août 1986 au 31 juillet 1987, 22,24 t de phosphore entrent dans la retenue, soit plus du triple de la charge "dangereuse". Selon le critère de Vollenweider, la retenue de Pareloup devrait donc avoir les caractéristiques d'un lac eutrophe. Cette conclusion correspond à celle de Fabre (1982) qui a également utilisé, entre autres, l'équation de Vollenweider pour définir le niveau trophique de 31 réservoirs français dont celui de Pareloup.

Cependant, bien que la charge annuelle en phosphore soit très nettement supérieure à la charge "dangereuse" définie par Vollenweider, le lac n'est pas eutrophe, la concentration moyenne mesurée dans la retenue n'étant que de $13 \mu \mathrm{g} \mathrm{P.I}{ }^{-1}$ en 86-87.

Le modèle de Canfield et Bachmann (1981) a été établi à partir de données obtenues pour 433 lacs artificiels. Ce modèle permet de prédire les concentrations en phosphore dans le lac à partir de la charge annuelle en phosphore. L'équation s'écrit :

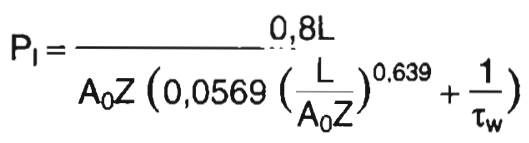

Pour une charge annuelle $(L)$ de 22,24 t la concentration attendue en phosphore dans le lac serait de

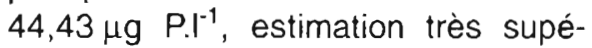
rieure à la concentration réelle mesurée $\left(13 \mu \mathrm{g} \mathrm{P.I^{-1 }}\right)$.

Le modèle OCDE (1982) a été établi à partir d'un programme OCDE sur l'eutrophisation piloté par R.A. Vollenweider en 1982. L'étude a porté sur 180 lacs et réservoirs artificiels situés en zone tempérée. Ce modèle permet d'obtenir la concentration en phosphore dans le lac $P_{1}$ à partir de la charge annuelle $\mathrm{L}$.

$$
P_{1}=1,55\left(\frac{L \tau_{w}}{Z A_{0}\left(1+v \tau_{w}\right)}\right)^{0,82}
$$

Pour une charge annuelle $(L)$ de $22,24 \mathrm{t}$, la concentration dans le lac prévue par ce modèle serait de $45,56 \mu \mathrm{g} \mathrm{P.} \mathrm{I}^{-1}$. Cette concentration est du même ordre que celle prévue avec le modèle de Canfield et Bachmann (1981) et est encore une fois très supérieure à la concentration réelle mesurée.

D'après la classification établie dans le rapport OCDE (1982), les concentrations calculées classent le lac de Pareloup dans la catégorie des lacs eutrophes, alors que les concentrations mesurées in situ le classent dans la catégorie des lacs mésotrophes. 
La différence entre les valeurs prédites par ces modèles et les valeurs mesurées pourrait avoir plusieurs origines:

- les hypothèses des modèles supposent que le lac est un réacteur homogène, qu'il n'y a pas de différences de concentrations dans la colonne d'eau, et que les concentrations des eaux de sortie sont égales à celles des eaux du lac. Autant d'hypothèses difficilement applicables à Pareloup.

- une des conditions d'application des modèles suppose des apports en phosphore constants au cours de l'année. Les apports irréguliers dus au pompage de Bage ne vérifient pas cette hypothèse.

- la diatomée Asterionella formosa semble jouer un rôle fondamental dans l'évolution de l'écosystème $\mathrm{Pa}$ reloup. Elle est abondante pendant la période d'isothermie de printemps, et n'étant pas consommée par le zooplancton, elle disparaît par sédimentation, ce qui immobilise dans le sédiment les nutriments qu'elle a consommés. Cette diatomée se comporte alors comme une "épuratrice" du phosphore (et de la silice) dans l'épilimnion (Thébault et Salençon, 1993; Salençon et Thébault, 1995). Les dosages de chlorophylle contenue dans les matières recueillies au printemps dans les pièges à sédiment et le rapport phosphore/chlorophylle du matériel en suspension pour cette même période ont permis de calculer que $66 \%$ à $100 \%$ du flux de phosphore vers les sédiments pendant la période printanière étaient at- tribuables à la sédimentation des diatomées (Labroue et al., 1994). Cette hypothèse est confirmée par le rapport OCDE (1982) qui souligne que la proportion de phosphore éliminé par sédimentation dans les lacs où dominent les diatomées est très supérieure à celle des lacs comptant davantage d'algues bleues ou bleues-vertes flottantes.

- les apports de Bage pénètrent au fond du lac près du barrage. Les campagnes de marquage (Salençon et Calmels, 1994) et les profils d'oxygène (Labroue et al., 1994) montrent qu'en période stratifiée (période de faible mélange), l'eau pompée reste confinée dans l'hypolimnion et évolue horizontalement en suivant la bathymétrie à une vitesse comprise entre 100 et $170 \mathrm{~m} \cdot \mathrm{h}^{-1}$. Le phosphore apporté par les pompages dans les couches profondes doit probablement sédimenter rapidement et être ainsi, en grande partie, éliminé du système.

\section{ESTIMATION DE LA SÉDIMENTATION}

\section{V.1 Méthodes d'estimation}

La sédimentation calculée est la différence entre le bilan des entrées et des sorties de phosphore et la variation journalière du stock de phosphore de la retenue:

$$
\mathrm{S}_{\text {calc }}=\mathrm{P}_{\text {in }}-\mathrm{P}_{\text {out }}-\Delta \mathrm{P}
$$

$\mathrm{S}_{\text {calc }}$ : sédimentation calculée

$P_{\text {in }}$ : quantité de phosphore entrant dans la retenue 
$P_{\text {out }}$ : quantité de phosphore sortant de la retenue

$\Delta \mathrm{P}$ : variation journalière du stock de phosphore de la retenue.

Celle-ci sera comparée avec les quantités de phosphore récoltées dans les pièges à sédiment.

Deux pièges à sédiment ont été immergés à $5 \mathrm{~m}$ du fond pendant une période s'étendant du 26 mars 1986 au 21 décembre 1987. Les durées d'immersion varient de dix à quarante jours. Les positions des pièges sont indiquées sur la figure 2. Les concentrations en phosphore sont dosées sur les sédiments déposés dans les pièges. Le taux de sédimentation a été évalué à partir de la moyenne des concentrations en phosphore du sédiment déposé dans les deux pièges.

\section{V.2 Résultats}

Pour une même période, les mesures effectuées dans les deux pièges à sédiment peuvent varier du simple au double. Nous avons pu cependant estimer à $19 \mathrm{t}$ la quantité totale de phosphore sédimentée sur toute la surface du plan d'eau. Celle-ci se répartit ainsi : 10,3t du 26 mars 1986 au 14 novembre 1986 et $8,5 \mathrm{t}$ du 12 mars 1987 au 21 décembre 1987. Ces valeurs sont donc sensiblement équivalentes pour les deux années.

Le flux de sédimentation mesuré varie, selon les périodes, de $3,93 \mathrm{mg} \cdot \mathrm{m}^{-2} \cdot \mathrm{j}^{-1}$ à $28,77 \mathrm{mg} \cdot \mathrm{m}^{-2} \cdot \mathrm{j}^{-1}$ avec une moyenne de $11,18 \mathrm{mg} \cdot \mathrm{m}^{-2} \cdot \mathrm{j}^{-1}$ (fig. 9). Les plus forts flux de sédimentation s'observent à l'automne, ils correspondent à la remise en suspen- sion des sédiments littoraux provoquée par la baisse de cote de la retenue et la turbulence apportée lors de la déstratification (Salençon et al., 1988).

La figure 10 compare les estimations de la sédimentation obtenues par le bilan entrées-sorties et par les pièges à sédiment. Ces deux approches restituent les mêmes variations saisonnières. II est regrettable que les données des pièges immergés en hiver n'aient pu être exploitées car d'après les estimations du bilan entrées-sorties la sédimentation semble importante.

Sur l'ensemble des périodes pour lesquelles nous disposons des deux types d'estimations, les quantités totales de phosphore sédimenté sont équivalentes (14,7 $t$ par le bilan et 13,4 t par les pièges). Par contre, les deux méthodes donnent des résultats différents si l'on compare les masses de phosphore sédimenté pour chaque durée d'immersion des pièges. D'avril à la mi-septembre, période de stratification, la sédimentation calculée à partir du bilan entrées-sorties est supérieure à la quantité de matériel récolté ce qui tendrait à confirmer l'hypothèse selon laquelle les apports de Bage sédimentent au pied du barrage et ne sont donc pas comptabilisées dans les pièges à sédiment. Par contre, vers la mi-mai et en automne, les quantités de phosphore récoltées sont supérieures aux quantités calculées. Cela correspond respectivement aux périodes de sédimentation des diatomées et de remise en suspension des sédiments littoraux (fig. 9). 


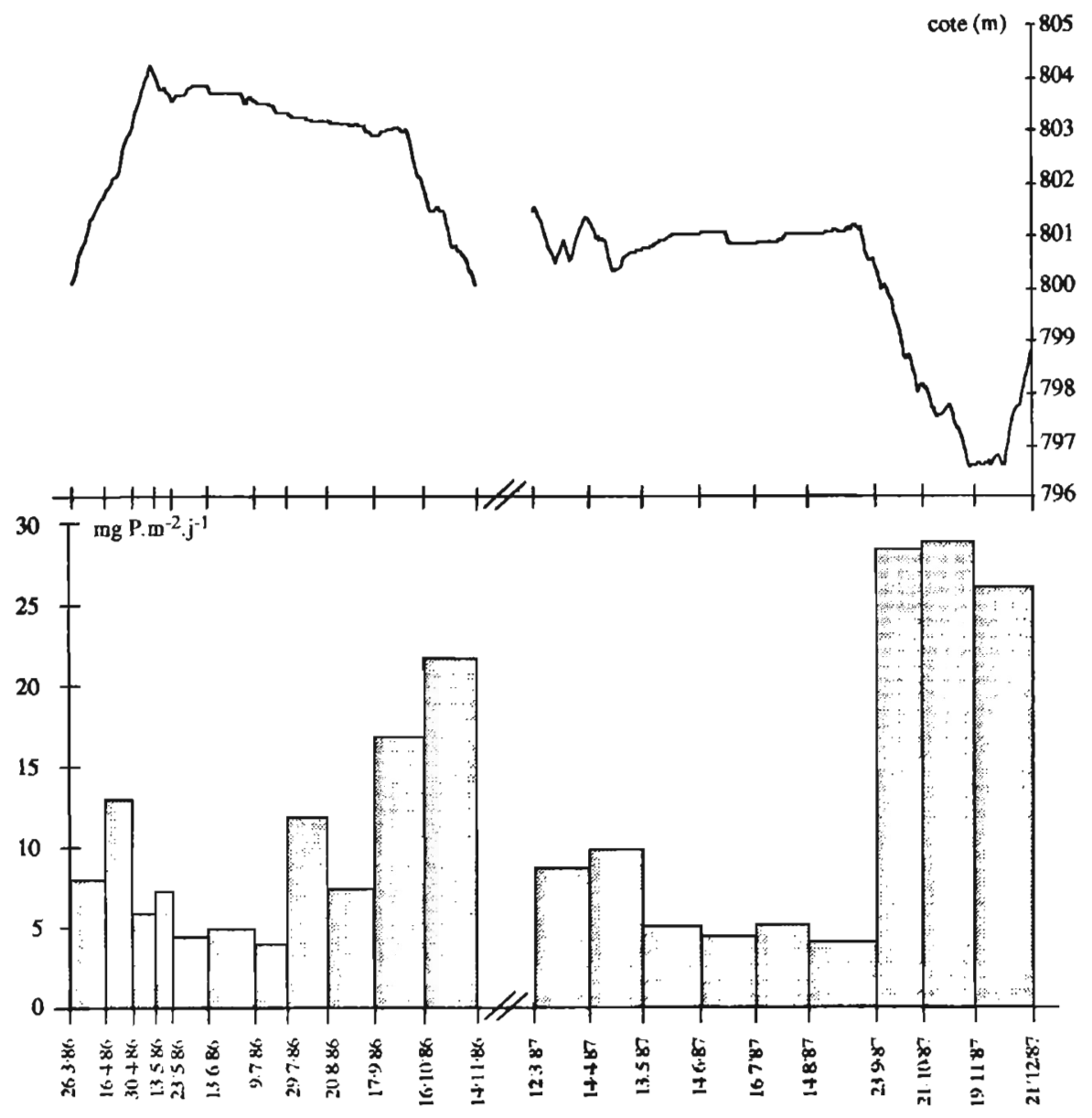

Fig. 9. - Comparaison de la cote du plan d'eau et des flux de sédimentation (calculés à partir des quantités de phosphore récoltées dans les pièges à sédiment).

Fig. 9. - Comparison of the reservoir level with sedimentation fluxes (calculated from amounts of phosphorus collected in sediment traps).

De plus, il ne faut pas oublier que les mesures des pièges à sédiment qui servent de référence à ce bilan présentent une grande variabilité, leur moyenne ne permet d'estimer que l'ordre de grandeur des quantités sédimentées.

\section{CONCLUSION}

Dresser un bilan du phosphore ne semble pas présenter, a priori, de grosses difficultés théoriques, surtout lorsqu'il exploite la banque de données de Pareloup, qui peut être consi- 


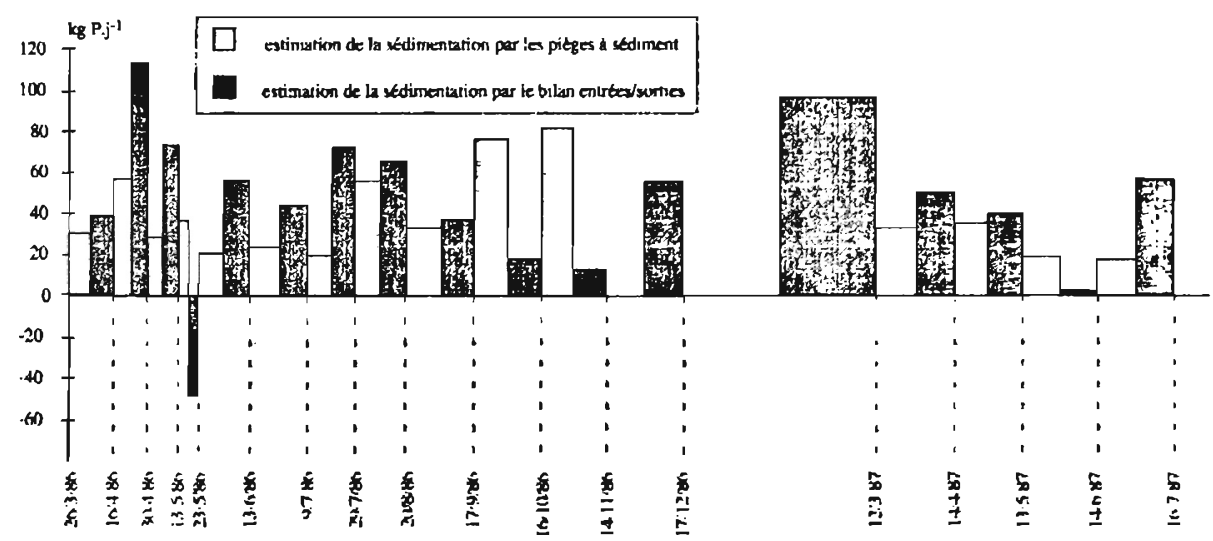

Fig. 10. - Comparaison entre les flux de phosphore sédimentés (calculés à partir du bilan d'entrées-sorties quotidien) et les flux déduits des mesures des pièges à sédiment pour l'ensemble du plan d'eau.

Fig. 10. - Comparison of sedimented phosphorus fluxes (calculated from the daily input-output budget) and fluxes deduced from measurement of sediment traps for the reservoir as a whole.

dérée, à juste titre, comme exceptionnelle aussi bien par la qualité que par l'abondance et la régularité des données. Nous avons, malgré tout, été confrontés à un manque d'homogénéité de ces données, essentiellement dû aux difficultés inhérentes aux mesures de terrain.

Il a alors été nécessaire de reconstituer les données manquantes par des interpolations et des régressions parfois hardies ou par des coefficients d'exportation issus de la littérature.

Avec la base de données constituée sur la retenue de Pareloup, le bilan du phosphore, calculé entre le $1^{\text {er }}$ avril 1986 et le 31 juillet 1987, estime à $29 \mathrm{t}$ les apports en phosphore dans la retenue. Ces apports proviennent en grande partie des bassins versants de Bage et du Viaur auxquels il convient d'ajouter ceux du bassin versant direct issus de la pol- lution domestique non négligeable durant l'été. Les apports du bassin versant de Pareloup ont lieu essentiellement lors des crues sous la forme de phosphore particulaire. Les crues les plus mobilisatrices sont celles qui ont lieu après des étiages prononcés, les matières en suspension ayant des teneurs en phosphore plus élevées qu'en période de hautes eaux.

Le faible niveau trophique du lac ne reflète pas les charges annuelles en phosphore: les modèles probabilistes de type Vollenweider ne sont pas applicables à Pareloup, d'une part, parce que la gestion hydraulique entraine des apports irréguliers de phosphore et, d'autre part, parce qu'une grande partie des apports en phosphore est piégée dans le sédiment, soit directement sous forme de MES, soit par sédimentation des diatomées printanières. 
Les quantités de phosphore sédimenté estimées par le bilan entréessorties d'une part, et par la récolte des pièges à sédiment d'autre part, concordent à l'échelle annuelle. A l'échelle saisonnière, il est par contre plus difficile d'estimer les quantités de phosphore disponibles pour la croissance du phytoplancton. Ces difficultés tiennent en particulier au comportement du phosphore lié aux MES véhiculées par les eaux affluentes et par les remises en suspension des sédiments littoraux. Au cours des développements ultérieurs, il serait donc intéressant d'évaluer la proportion de MES apportées et remises en suspension et de déterminer le pourcentage de phosphore biologiquement disponible liberé par ces MES au cours de leur séjour au sein de la zone pélagique.

Le fonctionnement hydraulique particulier de cette retenue ajoute à la complexité de l'estimation de son bilan de phosphore. Une grande partie des apports est assurée par le pompage des eaux de Bage qui proviennent de la retenue très eutrophe de Pont-de-Salars. Les campagnes de traçage des masses d'eau (Salençon et Calmels, 1994) ont déjà mis en évidence le cheminement des eaux de Bage dans l'hypolimnion, et certaines conditions dans lesquelles elles sont susceptibles de faire surface (upwellings dans les fonds de baies ou sur les hauts-fonds), favorisant alors la production primaire. Afin de préserver la qualité des eaux de Pareloup, il nous semble donc primordial de mieux connaître le devenir des eaux de Bage dans Pareloup au cours du cycle annuel.

\section{REMERCIEMENTS}

Nous tenons à remercier M. A. Fabre (CESF de Toulouse) et M. D. Mosnier (EDF-SEISO) pour les conseils et suggestions apportés lors de la rédaction de ce manuscrit.

\section{RÉFÉRENCES BIBLIOGRAPHIQUES}

Bosc P., 1985. Evaluation des apports en éléments nutritifs du bassin versant dans le lac de Pareloup (Aveyron). Mémoire de D.E.A., Université Paul Sabatier, Toulouse : 123 pp.

Calmels P. \& Salençon M.J., 1991. Techniques de marquage pour l'étude des circulations des masses d'eau dans les lacs. Application à la retenue de Pareloup. In: IAEA (Editor), Use of Isotope Techniques in Water Resources Development. Proc. Symp. 11-15 March 1991, Vienna. IAEAUUNESCO, Vienna, pp. 107-122.

Canfield D.E. Jr \& Bachman R.W., 1981. Prediction of total phosphorus concentrations, chlorophyll $a$, and Secchi depths in natural and artificial lakes. Can. J. Fish. Aquat. Sci., 38 : 414-423.

Dorioz J.M., Ferhi A., Pilleboue E., Dray M. \& Olive P., 1986. Sediment impact on phosphorus transport in a river system. Land use impacts on aquatic ecosystems. J. Lauga, H. Décamps and M.M. Holland ed. : 25-34.

Fabre A., 1982. Application d'un modèle de risque d'eutrophisation à un échantillon de résenvoirs. Compte-rendu de fin d'études de recherche financée par E.D.F., Direction de l'Equipement. Contrat $n^{\circ}$ E.2523, C.N.R.S., C.E.R.R., Toulouse $32 \mathrm{pp}$. 
Labroue L., Capblancq J., Salençon M.J., Toureng J.N. \& Mur C., 1994. Evolution saisonnière des éléments minéraux nutritifs $(P, N, S i)$ et de l'oxygène dissous dans le lac de Pareloup, Hydroécol. Appl. 6(1/2) : pp. 87-114.

Meybeck M., 1985. Méthodologie de l'établissement des bilans d'apports en éléments nutritifs aux systèmes lacustres. Rapport d'activité GRECO LACS, Laboratoire de Géologie E.N.S., Paris, $129 \mathrm{pp}$.

OCDE, 1982. Eutrophisation des eaux. Méthodes de surveillance, d'évaluation et de lutte. OCDE, Paris, $164 \mathrm{pp}$.

Rast W. \& Lee G.F., 1978. Summary analysis of the North American (U.S. portion) OECD eutrophication project: nutrient loading-lake response relationships and trophic state indices. EPA 600/3-78-008. U.S. EPA Corvalis Or. : 455 pp.

Salençon M.J., Bonnefille R. \& Klein P., 1976. Variabilité dans le temps des échanges océan-atmosphère: Influence des conditions météorologiques. Rapport HE31/76-26, Electricité de France, Paris, 93 pp.

Salençon M.J. \& Calmels P., 1994. Etude de la dynamique des masses d'eau du lac de Pareloup par traçages. Hydroécol. Appl, 6(1/2) : pp.19-58.

Salençon M.J. \& Thébault J.M., 1995. Simulation model of a mesotrophic reservoir (Lac de Pareloup): MELODIA, an ecosystem management reservoir model. Ecol. Modelling (sous presse).

Salençon M.J., Thébault J.M. \& Capblancq J., 1988. Elude de la retenue de Pareloup. Bilan des travaux rèalisés en 1987 dans le cadre de la Convention EDF-Ministère de l'Environnement. Rapport HE-31/88.23, Electricité de France, Paris, $43 \mathrm{pp}$.

Salençon M.J., Thébault J.M. \& Capblancq J., 1989. Etude de la retenue de Pareloup. Bilan des travaux réalisés en 1988 dans le cadre de la Convention EDF-Ministère de l'Environnement. Rapport HE-31/89.13, Electricité de France, Paris, 77 pp.

Thébault J.M. \& Salençon M.J., 1993. Simulation model of a mesotrophic reservoir (LaC de Pareloup): biological model. Ecol. Modelling, 65 : 1-30.

Vollenweider R.A., 1976. Advances in defining critical loading levels for phosphorus in lake eutrophication. Mem. Ist. Ital. Idrobiol., 33 : 53-83. 